\title{
Intracerebral transplantation of mesenchymal stem cells into acid sphingomyelinase-deficient mice delays the onset of neurological abnormalities and extends their life span
}

\author{
Hee Kyung Jin, ${ }^{1}$ Janet E. Carter, ${ }^{2}$ George W. Huntley, ${ }^{3}$ and Edward H. Schuchman ${ }^{1,4}$ \\ ${ }^{1}$ Department of Human Genetics, Mount Sinai School of Medicine, New York, New York, USA \\ ${ }^{2}$ Department of Neuroscience and Old Age Psychiatry Institute of Psychiatry, London, United Kingdom \\ ${ }^{3}$ Fishberg Center for Neurobiology, Mount Sinai School of Medicine, New York, New York, USA \\ ${ }^{4}$ Carl C. Icahn Institute for Gene Therapy and Molecular Medicine, Mount Sinai School of Medicine, \\ New York, New York, USA \\ Address correspondence to: Edward H. Schuchman, Department of Human Genetics, Mount Sinai School of Medicine, \\ 1425 Madison Avenue, Room 14-20A, New York, New York 10029, USA. \\ Phone: (212) 659-6711; Fax: (212) 849-2447; E-mail: Edward.Schuchman@mssm.edu.
}

Received for publication December 14, 2001, and accepted in revised form March 25, 2002.

Types A and B Niemann-Pick disease (NPD) are lysosomal storage disorders resulting from loss of acid
sphingomyelinase (ASM) activity. We have used a knockout mouse model of NPD (ASMKO mice) to
evaluate the effects of direct intracerebral transplantation of bone marrow-derived mesenchymal stem
cells (MSCs) on the progression of neurological disease in this disorder. MSCs were transduced with
a retroviral vector to overexpress ASM and were injected into the hippocampus and cerebellum of
3-week-old ASMKO pups. Transplanted cells migrated away from the injection sites and survived at
least 6 months after transplantation. Seven of 8 treated mice, but none of the untreated controls, sur-
vived for $\geq 7$ months after transplant. Survival times were greater in sex-matched than in sex-mis-
matched transplants. Transplantation significantly delayed the Purkinje cell loss that is characteris-
tic of NPD, although the protective effect declined with distance from the injection site. Overall ASM
activity in brain homogenates was low, but surviving Purkinje cells contained the retrovirally expressed
human enzyme, and transplanted animals showed a reduction in cerebral sphingomyelin. These
results reveal the potential of treating neurodegenerative lysosomal storage disorders by intracerebral
transplantation of bone marrow-derived MSCs.

J. Clin. Invest. 109:1183-1191 (2002). DOI:10.1172/JCI200214862.

\section{Introduction}

Types A and B Niemann-Pick disease (NPD) are lysosomal storage disorders resulting from the deficient activity of acid sphingomyelinase (ASM; EC 3.2.1.14). Both forms of the disorder are characterized by progressive lipid accumulation, foam cell infiltration, and organomegaly, but whereas type B NPD patients have little neurological involvement and can survive into adulthood, type A NPD patients follow a rapid neurodegenerative course resulting in early childhood death (1). The ASM knockout mouse (ASMKO mouse) provides a model in which to study the pathogenesis and treatment of both forms of NPD. The phenotype of this mouse model includes absent ASM activity, progressive lipid accumulation in various organs, ataxia, and Purkinje cell loss $(2,3)$.

Previous work from our laboratory has shown that intravenous transplantation of ASM-expressing bone marrow cells can effectively prevent or reverse the visceral organ pathology in ASMKO mice by providing bone marrow-derived cells that can release ASM for uptake by NPD cells $(4,5)$. However, only a modest impact on CNS disease occurred from these treatments, most likely due to limited engraftment of donor-derived cells in the brain.
Mesenchymal stem cells (MSCs) are stem cell-like precursors found in the bone marrow that can produce nonhematopoietic cells such as osteoblasts, chondrocytes, adipocytes, and myoblasts, and act as support cells that are important in the differentiation and growth of hematopoietic stem cells (6). Systemic infusion of MSCs into irradiated mice has previously been shown to produce donor-derived cells in a variety of nonhematopoietic tissues, including the brain (7). Of direct relevance to this study, a recent study has also found that following systemic infusion, donor-derived cells can cross the blood-brain barrier and differentiate into Purkinje cell neurons (8), providing a further indication of the multipotential nature of these cells. In addition, direct injection of human MSCs into the brain parenchyma has resulted in engraftment of donor-derived cells, and importantly, the transplanted MSCs rapidly moved away from the injection sites along known neural stem cell migratory pathways (9-11). Small numbers of these cells also differentiated into neural cell types. Similar results have been obtained following intraventricular injection (9).

The goal of the current study was to evaluate whether the transplantation of retrovirally transduced MSCs 
overexpressing human ASM could evoke a therapeutic effect in the CNS of ASMKO mice. We present data that shows that injection of 100,000 cells into the brain parenchyma extends the life span of the treated mice, and that this could be correlated with a significant reduction in the rate of Purkinje cell loss and improved cerebellar function. Since the source of injected MSCs was transgenic mice expressing green fluorescent protein (GFP), we were also able to monitor the migration and persistence of these cells following transplantation. The results described here provide a rationale for the further investigation of these cells for the treatment of diseases with widespread neuropathology, such as the neurodegenerative lysosomal storage disorders.

\section{Methods}

Animals. The ASMKO mouse model was created by gene targeting as described previously (2). Affected mice have no detectable ASM activity, but develop normally until about 8 weeks of age, when ataxia and mild tremors become noticeable. The disease then follows a neurodegenerative course that leads to death between 6 and 8 months of age. Characteristic lipid-laden foam cells (NPD cells) are found in most major organs and are associated with elevated sphingomyelin levels. Affected (homozygous) mice are distinguished from normal animals using a PCR-based assay (2). The animals were maintained on a 12-hour light/dark cycle and given water ad libitum and Purina rodent chow 5001.

Isolation and culture of MSCs. To obtain murine MSCs, tibias and femurs were dissected from 6- to 8-week-old homozygous (GFPU)5Nagy male mice (12) (The Jackson Laboratory, Bar Harbor, Maine, USA). Bone marrow was harvested by flushing the medullary cavities with $5 \mathrm{ml}$ of buffered Hank's solution $(10 \mu \mathrm{mol} / \mathrm{ml}$ HEPES, pH 7.5) using a 26-gauge needle. Single-cell suspensions were obtained by passing the cells through a $40-\mu \mathrm{m}$ cell strainer (Becton-Dickinson and Co., Franklin Lakes, New Jersey, USA), and approximately $10^{7}$ cells were plated in a $75-\mathrm{cm}^{2}$ flask containing $10 \mathrm{ml}$ of MyeloCult M5300 long-term bone marrow culture medium (StemCell Technologies Inc., Vancouver, Canada) supplemented with freshly prepared hydroxycortisone hemisuccinate $(1 \mu \mathrm{M}$; Sigma Chemical Co., St. Louis, Missouri, USA), $100 \mathrm{IU} / \mathrm{ml}$ penicillin, $100 \mu \mathrm{g} / \mathrm{ml}$ streptomycin, and $250 \mathrm{ng} / \mathrm{ml}$ amphotericin B (Invitrogen Corp. Carlsbad, California, USA). The cell cultures were grown for 2 weeks, and the plastic-adherent cell population (i.e., MSCs) was used for subsequent experiments.

Retroviral transduction of MSCs. The ASM/MFG retroviral vector was constructed by inserting the fulllength human ASM cDNA (hASM) into the MFG vector as previously described (13). To achieve retroviral transduction, after 2 weeks in culture, the bone marrow cell medium was changed and the plastic-adherent cells (MSCs) were cultured for an additional 72 hours in $10 \mathrm{ml}$ of DMEM containing the ecotropic hASM/MFG virus (titer, approximately $5 \times 10^{5} / \mathrm{ml}$ ), $10 \%$ heat-inactivated FCS, and antibiotics. ASM activ- ity in the culture media of the transduced and nontransduced cells and in brain homogenates (see below) was determined as previously described $(2,13)$.

Transplantation of the transduced cells. ASMKO mice (approximately 3 weeks of age, $n=20$ ) were anesthetized and placed on the Styrofoam platform of a stereotaxic injection apparatus (David Kopf Instruments, Tujunga, California, USA). Animals were anesthetized with $0.02 \mathrm{ml} / \mathrm{g}$ body weight of (2,2,2-tribromoethanol and 2-methyl-2-butanol; Sigma Chemical Co.). The skull was exposed by a $15-\mathrm{mm}$ incision in the midline, and two small skull flaps were raised using a fine-gauge needle. The injection coordinates were determined in pilot studies by injecting fast green dye into normal adult mouse brains and locating the injection position in sectioned brains. The final injection coordinates for the hippocampus were $2.06 \mathrm{~mm}$ posterior to bregma and $2.00 \mathrm{~mm}$ mediolateral, and the injections were carried out at a depth of $2.0 \mathrm{~mm}$. For the cerebellum, the injection coordinates were $5.52 \mathrm{~mm}$ posterior to bregma and the injection depth was 2.50 $\mathrm{mm}$. (Figure 7a schematically shows the sites of injection and the sections that were used for Purkinje cell quantitation.) Each injection was $1 \mu \mathrm{l}$ and contained approximately 50,000 cells. The injections were carried out using a glass capillary $(1.2 \mathrm{~mm} \times 0.6 \mathrm{~mm})$ pulled to a fine tip and attached to a Hamilton syringe. The injections were delivered at a rate of $0.05 \mu \mathrm{l} / \mathrm{min}$, and the needle was slowly withdrawn after an additional 2 minutes. The scalp was closed by suture and the animals recovered from the anesthesia with gentle warming under an infrared heat lamp before they were returned to their mothers. Age-matched affected (but not injected) and normal littermates were used as controls ( $n=20$ for each group).

Tissue processing. At 4, 12, and 24 weeks after transplantation, injected animals and control littermates were anesthetized with Avertin and sacrificed by cardiac perfusion. The left ventricle was cannulated, an incision was made in the right atrium, and the animals were perfused with 4\% paraformaldehyde in PBS until the outflow ran clear. To visualize GFP-expressing cells, brains were postfixed in $10 \%$ formalin for at least 24 hours. Fifty-micron coronal sections were cut using a vibratome, and freefloating serial sections were collected into PBS. The sections were mounted in Vectashield with 4,6-diamidino2-phenylindole (DAPI; Vector Laboratories Inc., Burlingame, California, USA) to counterstain the cell nuclei, and GFP-expressing cells were identified by their blue-green appearance using a Nikon Eclipse E800 microscope. For calbindin immunohistochemistry (see below), brains were postfixed in 10\% formalin for 24 hours and cryoprotected in 30\% sucrose. Tissues were cut into $15-\mu \mathrm{m}$ coronal sections using a cryostat. The sections were adhered to Superfrost Plus coated glass slides (Fisher Scientific Co., Pittsburgh, Pennsylvania, USA) and stored at $-20^{\circ} \mathrm{C}$ until use.

Purkinje cell quantitation. Frozen sections were treated with blocking buffer (PBS, pH 7.4, containing $20 \%$ goat 
serum) for 30 minutes and then labeled with rabbit anti-calbindin polyclonal antibodies (Chemicon International Inc., Temecula, California) (1:500 dilution in blocking buffer) for 3 days at $4^{\circ} \mathrm{C}$. The tissue sections were washed in PBS and incubated with rabbit IgG biotinylated secondary antibodies (Vectastain ABC kit; Vector Laboratories Inc.) overnight at $4^{\circ} \mathrm{C}$, and then transferred to an avidin-biotin complex for a 30-minute incubation. After three washes with PBS, the sections were developed in $0.04 \%$ hydrogen peroxide and $0.05 \%$ 3,3-diaminobenzidine (DAB; Vector Laboratories Inc.) for 10 minutes. Nuclei were counterstained by mounting sections under $1.5-\mathrm{mm}$-thick coverslips in Permount (Fisher Scientific Co., Morris Plains, New Jersey, USA). For each injected or control animal, at least 25 sections were analyzed using a Nikon Eclipse E800 fluorescent microscope. To quantify the number of Purkinje cells, the images from individual sections were saved in Photoshop version 5.0 (Adobe Systems Inc., San Jose, California, USA). The Photoshop images were magnified to the same degree for each section and printed, and the number of Purkinje cells was counted from the prints and expressed as cell number/inch.

Immunostaining using anti-buman ASM antibodies. Frozen brain sections were treated with blocking buffer (PBS, pH 7.4, containing 20\% goat serum) for 30 minutes and then labeled with rabbit anti-human ASM IgG polyclonal antibodies (14) (1:500 dilution in blocking buffer) for 1 day at $4^{\circ} \mathrm{C}$. The sections were then washed in PBS and incubated with rabbit IgG biotinylated secondary antibodies (Vectastain ABC kit; Vector Laboratories Inc.) for 2 hours at room temperature, and then transferred to a solution containing avidinbiotin complex for 30 minutes. After three washes with PBS, the sections were developed in $0.04 \%$ hydrogen peroxide and $0.05 \% \mathrm{DAB}$ (Vector Laboratories Inc.) for 10 minutes. Nuclei were counterstained by mounting sections under $1.5-\mathrm{mm}$-thick coverslips in Permount. The sections were then analyzed using a Nikon Eclipse E800 fluorescent microscope.

Lysenin immunostaining. Frozen sections were treated with blocking buffer consisting of PBS, pH 7.4, containing 1\% (wt/vol) BSA for 1 hour. The slides were then incubated with $1 \mu \mathrm{g} / \mathrm{ml}$ lysenin, a sphingomyelin-spe- cific binding protein (15), in 1\% BSA-PBS for 2 hours, washed with $1 \%$ BSA-PBS, and then incubated with anti-lysenin antiserum (1:1,000 dilution in blocking buffer) for 1 hour. The slides were washed in 1\% BSAPBS and incubated with Alexa Fluor 568-conjugated anti-rabbit IgG (Molecular Probes Inc., Eugene, Oregon, USA) for 1 hour. Nuclei were counterstained by mounting sections under 1.5 -mm-thick coverslips in Vectashield with DAPI. The sections were then analyzed using a Nikon Eclipse E800 fluorescent microscope.

Accelerating rotarod analysis. The rotarod test assesses an animal's balance and coordination by measuring the amount of time the animal is able to remain on a longitudinally rotating rod. The rotarod apparatus (accelerating model 7650; Ugo Basile Biological Research Apparatus, Comerio, Italy) (16) has a 3-cm-diameter rod, suitably machined to provide grip. In this apparatus, a motor sets the rotor in motion via the gear belt at a selected speed. When the mouse falls off its cylinder section, the plate below trips and the corresponding counter is disconnected, thereby recording the animal's endurance time in seconds. Use of an accelerating model ensures that screening results are less scattered (17). The machine was set to an initial speed of $32 \mathrm{rpm}$, and the acceleration was increased by $32 \mathrm{rpm}$ every 25-30 seconds. Treated ASMKO mice were analyzed along with untreated ASMKO and normal control animals. Scores were registered every week (at least two independent tests were performed for each timepoint) beginning at 12 weeks after injection. Uniform conditions were carefully maintained for each test, and there was a rest time of 1 hour between trials. A maximum time limit of $300 \mathrm{~s} /$ test was established. Data were analyzed using a one-tailed Student $t$ test, and comparisons were made between the treated and control groups.

\section{Results}

In vivo MSC survival and anatomical distribution. In an initial pilot experiment, approximately 50,000 MSCs were obtained from GFP transgenic mice and transplanted unilaterally into the hippocampus (dentate gyrus) of 1to 2-day-old ASMKO mice. Animals were sacrificed 4 weeks after transplantation, and the GFP-expressing cells were located in brain sections. As shown in Figure a

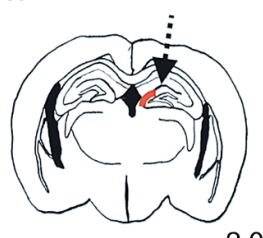

b

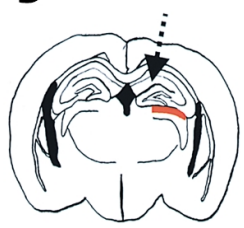

$-2.06$
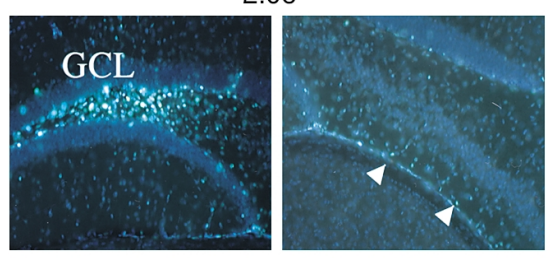

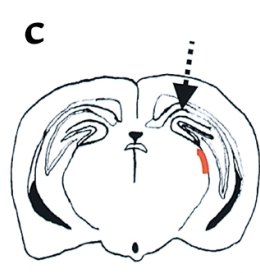

$-2.70$

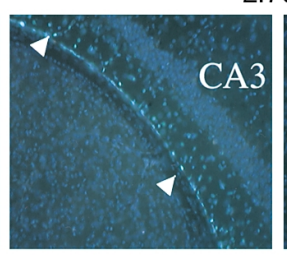

d
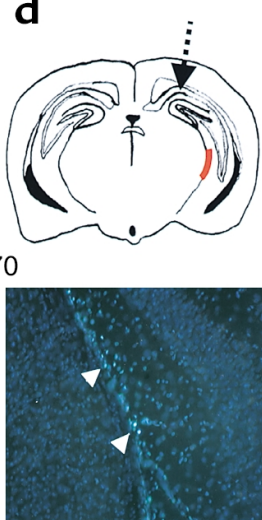

Figure 1

Survival and migration of MSCs in the ASMKO mouse brain 1 month after injection. Above each micrograph is a schematic depiction of the brain indicating the position of the injection site (dotted arrows) and the area shown in the photographs below (highlighted in red). Arrowheads indicate blue-green nuclei of transplanted GFP-expressing MSCs counterstained with DAPI. (a) Clustering of MSCs at the dentate gyrus. (b) Lateral migration, at a position 2.06 $\mathrm{mm}$ posterior to bregma. (c and d) Lateral migration of MSCs $2.70 \mathrm{~mm}$ posterior to bregma. GCL, granule cell layer; CA3, CA3 region of the hippocampus. Original magnification $\times 20$. 


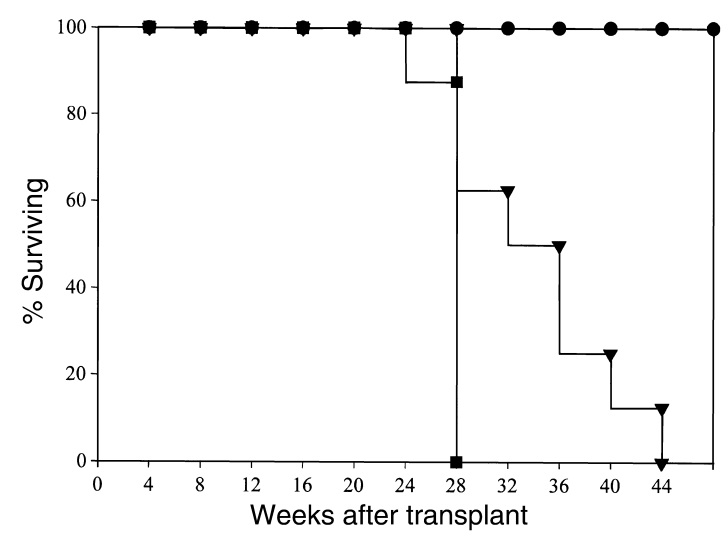

Figure 2

Survival of ASMKO mice receiving intracerebral MSC transplants. By 28 weeks after transplant (31 weeks of age), all of the untreated ASMKO mice (squares) had died, whereas seven of eight treated ASMKO animals (triangles) and all of the normal animals (circles) survived. The longest-surviving treated ASMKO mouse lived to be about 47 weeks old ( 44 weeks after transplant). $n=8$ for each group.

1a, the largest number of GFP-positive cells could be identified in clusters just below the granule cell layer of the hippocampus, close to the injection site. This clustering phenomenon has previously been identified as characteristic for cells injected at this site, due to the presence of a cleavage plane beneath the granule cell layer $(18,19)$. A significant number of transplanted cells had also migrated from the injection site by 4 weeks, either laterally along the external capsule (not shown) or medially beneath the CA3 region of the hippocampus (Figure 1, b-d).

In all transplanted animals, GFP-expressing cells were identified in multiple areas of the brain beyond the injection site, including the contralateral cortex, the ependymal lining of the lateral ventricles, and the corpus callosum, and were frequently found adjacent to blood vessels. The largest numbers of migrating cells were seen along a rostrocaudal axis ipsilateral to the injection site, covering a distance of approximately 900 $\mu \mathrm{m}$ and migrating in an organized fashion in parallel arrays along white matter tracts toward the lateral ventricles. These observations were similar to those reported previously (9-11).

Survival and growth of transplanted ASMKO mice. Based on the excellent migratory capacity of the transplanted MSCs observed in our pilot experiment and by other investigators, 20 additional 3-week-old ASMKO mice received two transplants of 50,000 cells each into the hippocampus and cerebellum, respectively (see Figure 7a for a schematic depiction of the injection sites). Three-weekold animals were used for this study because at this age all of the treated animals could survive the double transplant procedure. In addition, 3-week-old ASMKO animals do not yet exhibit any neurological deficits (2). To maximize therapeutic effects, the MSCs from the GFP transgenic mice were transduced with a retroviral vector expressing human ASM. Prior to transplantation these cells released up to 30 -fold more ASM activity into the culture media than did nontransduced MSCs (not shown). Previous studies have shown that ASM released by transduced cells can be taken up by many different cell types, including neurons (5).

Of the 20 treated animals, eight were maintained in our animal facilities for up to 47 weeks; animals in this survival group were analyzed on a weekly basis. The other animals were sacrificed at various timepoints for histological and biochemical analyses. By 28 weeks after transplant, all of the untreated ASMKO control mice had died, consistent with previous published reports concerning the life span of these animals (Figure 2). In contrast, seven of eight treated ASMKO animals were alive. At 36 weeks after transplant, four of eight treated animals were still alive, and one survived for 44 weeks after transplant. Of the four animals surviving beyond 36 weeks, three were male and one was female.

We also monitored the body weights of the survival group and their performance on an accelerating
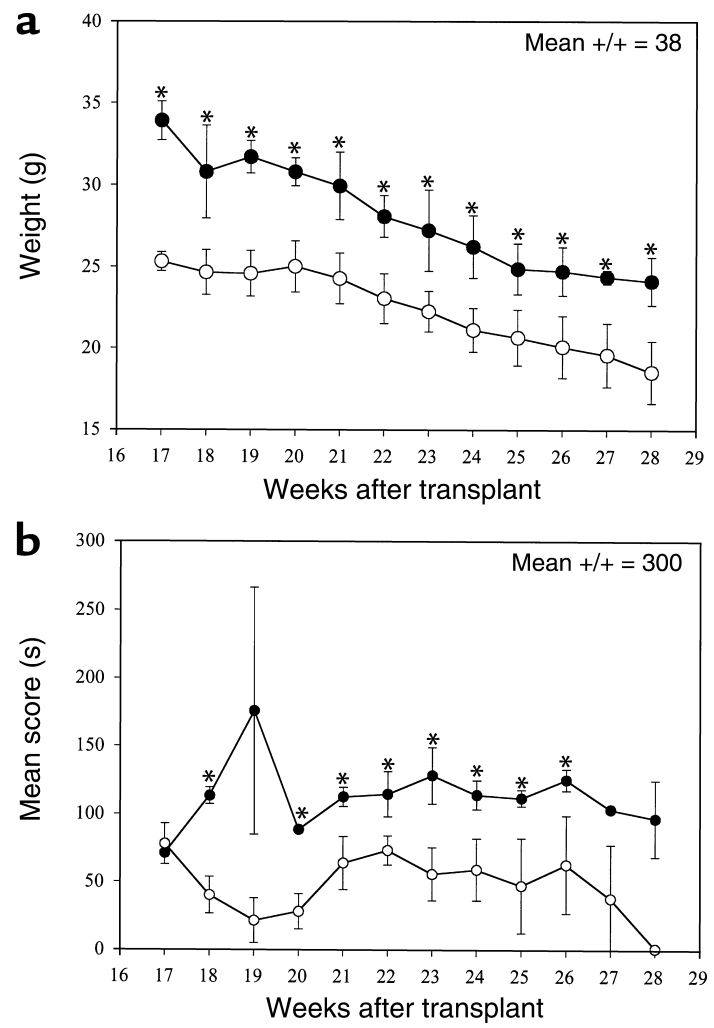

\section{Figure 3}

Body weights and rotarod scores for treated and untreated male ASMKO mice. (a) The mean body weights of treated (filled circles) and untreated (open circles) ASMKO male mice $(n=4)$ are shown beginning at 17 weeks after transplant (20 weeks of age). The mean body weight ( $38 \mathrm{~g}$ ) of normal male animals $(+/+)$ is also indicated. (b) Rotarod scores of the same treated (filled circles) and untreated (open circles) male mice were averaged and plotted beginning 17 weeks after transplant (20 weeks of age). The mean rotarod score $(300 \mathrm{~s})$ of normal male animals $(+/+)$ is indicated. All of the untreated control animals died by 28 weeks after transplant (see Figure 2 ). ${ }^{*} P<0.05$, treated versus untreated, per one-tailed Student $t$ test. 

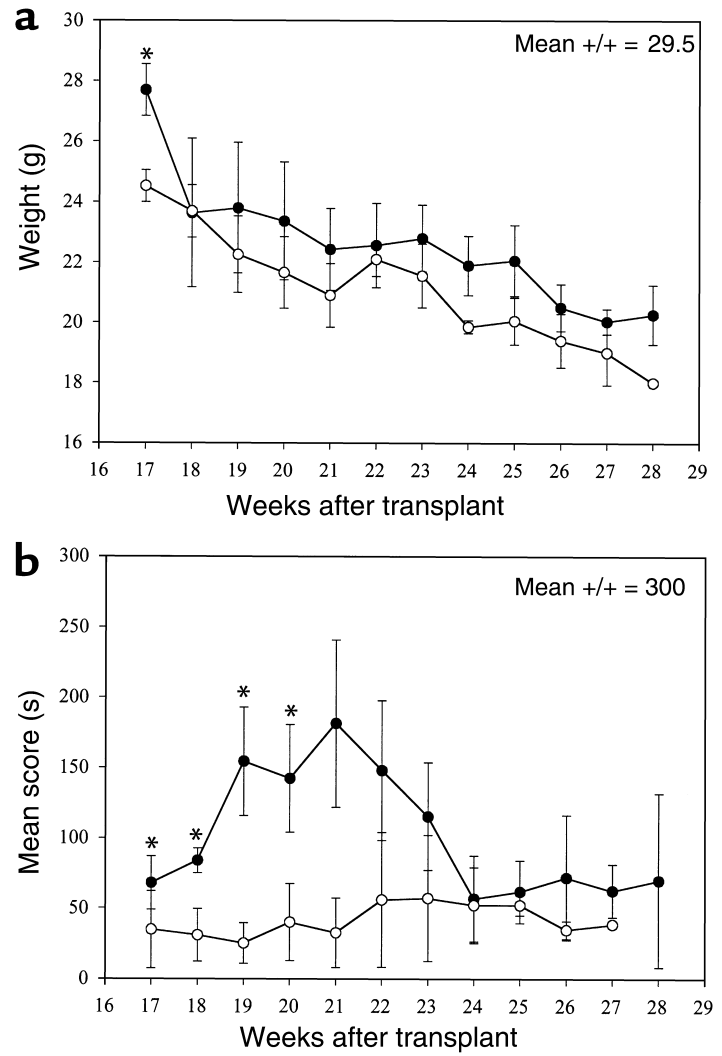

Figure 4

Body weights and rotarod scores for treated and untreated female ASMKO mice. (a) The mean body weights of treated (filled circles) and untreated (open circles) ASMKO female mice $(n=3)$ are shown beginning at 17 weeks after transplant (20 weeks of age). The mean body weight $(29.5 \mathrm{~g})$ of normal female animals $(+/+)$ is also indicated. (b) Rotarod scores of individual female treated (filled circles) and untreated (open circles) mice were averaged and plotted beginning 17 weeks after transplant ( 20 weeks of age). The mean rotarod score (300 s) of normal female animals $(+/+)$ is indicated. All of the untreated animals died by 28 weeks after transplant (see Figure 2 ). ${ }^{*} P<0.05$, treated versus untreated, per one-tailed Student $t$ test.

rotarod. Figure 3 shows the data for male mice (data is graphically displayed until 28 weeks after transplant, at which time all of the untreated ASMKO control animals had died). Treated ASMKO male mice had significantly higher body weight (Figure 3a) and significantly better rotarod performance scores than did the untreated ASMKO mice (Figure $3 \mathrm{~b}$ ). Note however that the maximal rotarod endurance time of the treatment group was only about one third of normal, and that the weight of the mice in this group began to decline by 20 weeks after transplant. Less significant results were obtained with the female recipients, and only one survived beyond 32 weeks (Figure 4). Since the donor cells were obtained from male animals (see Methods), we assume that this difference between male and female recipients was due to immunological rejection of the donor cells in the brains of the females. Indeed, analysis of GFPexpressing cells in the female transplant recipients at
8 weeks after transplant showed fewer donor cells than were present in male brains (not shown).

Quantitative analysis of Purkinje cells in the cerebellum after transplantation. A characteristic feature of the ASMKO mouse phenotype is the progressive degeneration of Purkinje cells within the cerebellum, together with widespread cytoplasmic vacuolar storage in neurons throughout the cerebral cortex (1). Purkinje cells were quantified by immunohistochemistry using calbindin antibodies. Figure 5 shows representative sections from treated ASMKO and control (untreated ASMKO and normal) mice at 4, 12, and 24 weeks after transplant. For each animal, the same section is shown, $75 \mu \mathrm{m}$ from the cerebellar injection site (position A in Figure $7 \mathrm{a}$ ). Note that by 12 weeks after transplant, an almost complete disappearance of Purkinje cells was evident in the untreated ASMKO mice, and there was extensive vacuolization. In contrast, the Purkinje cell number and vacuolization in treated ASMKO mice was marked-

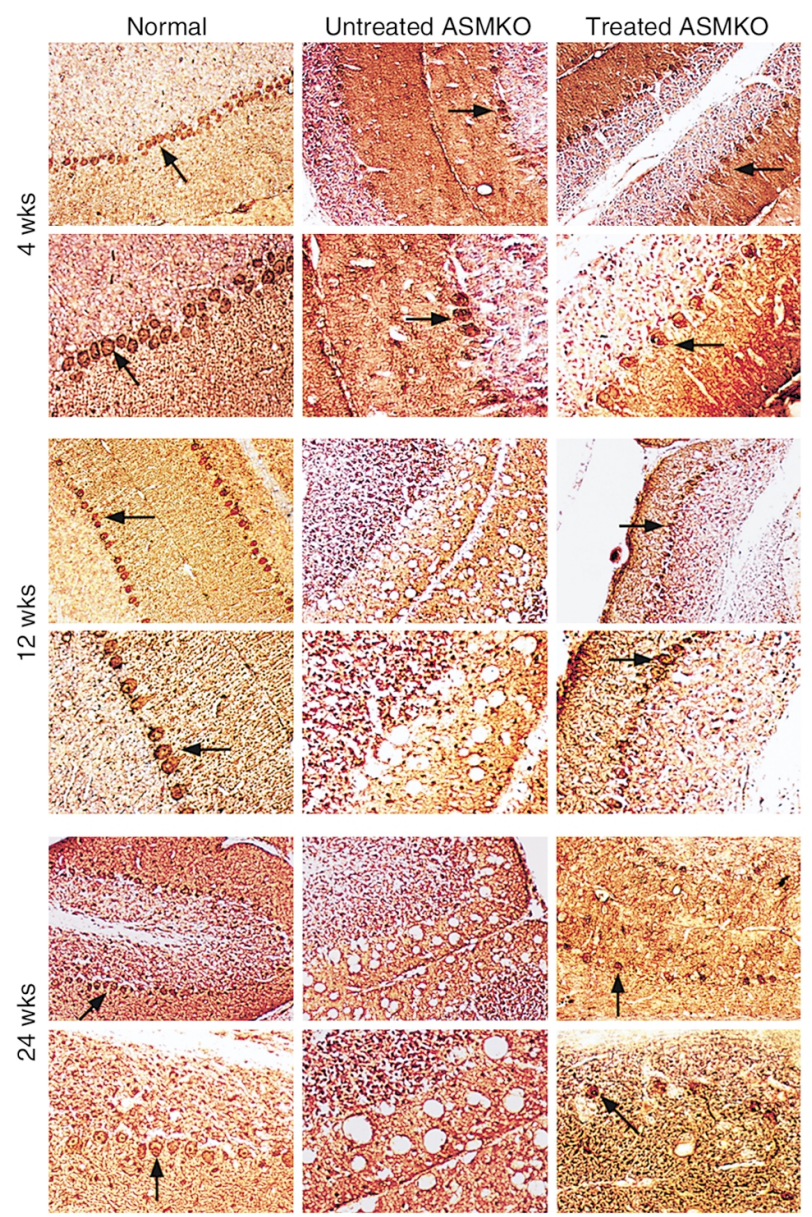

\section{Figure 5}

Purkinje cell immunohistochemistry at 4, 12, and 24 weeks after transplantation. Visualization of Purkinje cells was performed using anticalbindin antibodies as described in Methods. Arrows indicate Purkinje cells. Note the extensive vacuolization and absence of Purkinje cells in the untreated ASMKO mice at 12 and 24 weeks after transplant, compared with the treated animals. The sections shown are from position $\mathrm{A}$ in Figure $7 \mathrm{a}, 75 \mu \mathrm{m}$ from the cerebellar injection site. 


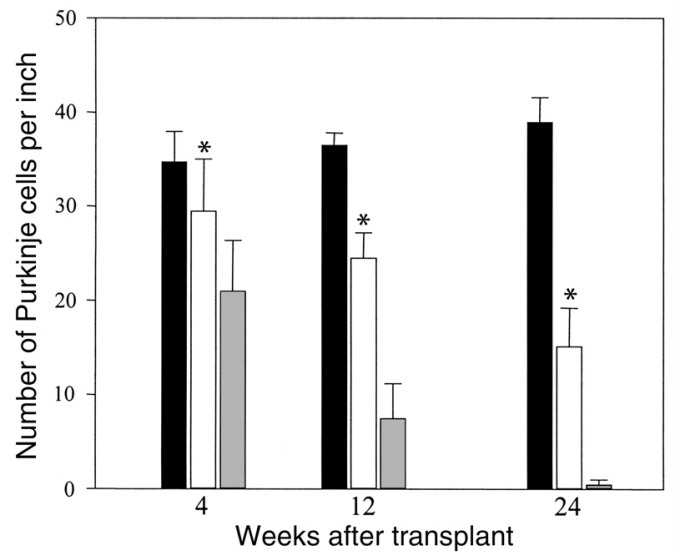

Figure 6

Histograms showing Purkinje cell numbers in treated ASMKO mice at 4, 12, and 24 weeks after transplantation. Quantitation was performed as described in Methods. The data shown in the histograms correspond to the images shown in Figure 5 (position A in Figure 7a). Data are expressed as mean $\pm \operatorname{SEM}(n=4)$. Black bars indicate normal control animals, white bars indicate treated ASMKO animals, and gray bars indicate untreated ASMKO control animals. ${ }^{*} P<0.05$, treated versus untreated ASMKO mice, per one-tailed Student $t$ test.

ly improved. By 24 weeks the treated animals were still improved, although some cell loss had occurred. Graphical representation of the data from these slides is shown in Figure 6, confirming a significant reduction in the loss of Purkinje cells at 4,12, and 24 weeks after transplantation in the treatment group $(P<0.05$, treated versus untreated groups). Importantly, the protective effect of the transplant procedure on the Purkinje cell numbers was a function of distance from the cerebellar injection site, as shown in Figure 7. Position A in this figure corresponds to the micrographs shown in Figure 5. Note that 12 weeks and 24 weeks after transplant (black squares and triangles), sections that were further away from the injection site had fewer surviving Purkinje cells.

Analysis of human ASM expression and sphingomyelin levels in the cerebellum of treated mice. In an attempt to correlate the clinical and pathological findings with increased ASM activities, we first attempted to detect activity in total brain homogenates from the treated animals. However, the levels of activity were below detectable limits ( $<1 \%$ of normal) (not shown), consistent with the small number of injected cells. We next used anti-human ASM antibodies to detect the expressed enzyme in cerebellar sections. As shown in Figure 8a, these antibodies did not cross-react with murine ASM in brain sections from normal mice, but did detect a specific signal in surviving Purkinje cells of the treated animals. We also used lysenin, a sphingomyelin-specific binding protein, to assess sphingomyelin storage in the cerebellar sections from treated animals. As shown in Figure 8b, the amount of lysenin staining in MSC-injected mice was markedly decreased compared with that in tissues from untreated ASMKO animals.

\section{Discussion}

In the present study, the suitability of MSCs as enzyme delivery vehicles for treatment of neurological lysosomal storage diseases was evaluated using the ASMKO mouse model of NPD. To effectively treat such diseases, a delivery vehicle is required that can migrate throughout the brain, survive in vivo long-term, and release normal enzymes for uptake by surrounding neurons and other neural cells. Major advantages of MSCs include the facts that they can be easily obtained from affected patients, are readily transduced with gene transfer vectors, and the transduced cells secrete catalytically active enzymes that can be taken up by a variety of cell types, including neurons $(20,21)$. In addition, previous studies in animal models have shown that MSCs can be transplanted into the brain, migrate throughout the CNS, and survive for several months $(9,10)$. There is also very recent in vivo evidence demonstrating that transplanted bone marrow-derived cells can differentiate into Purkinje cell neurons, a cell type that is affected in type A NPD and other neurodegenerative lysosomal storage disorders (8).

We obtained MSCs from the bone marrow of adult GFP transgenic mice using the method of plastic adherence (11). This relatively crude procedure produces a heterogeneous population, including multipotential MSCs that can be readily differentiated into osteoblasts, adipocytes, chondrocytes, and myoblasts (22-25). The use of GFP-expressing MSCs provided a unique detection system facilitating the identification of transplanted cells. Although alternative protocols are available for the isolation of more homogeneous MSC cultures, e.g., by removing lymphohematopoietic cells (10), it has not been established that removal of these cells confers a significant advantage for survival and/or migration in the CNS, nor has it been established whether such procedures deplete the MSC population of multipotential precursors. Therefore, for our initial studies we chose to use this crude method of MSC preparation in order to avoid depletion of potentially important cells.

The results presented here demonstrate that the transplantation of MSCs into the CNS of ASMKO mice had a protective effect on Purkinje cells and significantly extended the life span of treated mice compared with untreated ASMKO control animals. In addition, the cerebellar function of the treated animals (as quantified using an accelerating rotarod apparatus) was significantly improved. It is notable that these results were obtained despite the fact that no detectable ASM activity was found in brain homogenates at any time after transplant. This is consistent with previous bone marrow transplantation (4) and hematopoietic stem cell gene therapy (5) studies in which the life span, Purkinje cell survival, and cerebellar function of the treated animals was similarly improved despite undetectable ASM levels in the brain. In contrast, intravenous infusion of recombinant ASM had no effect on these neurological parameters (26), suggesting that the positive results we observed here and in our previous 
a
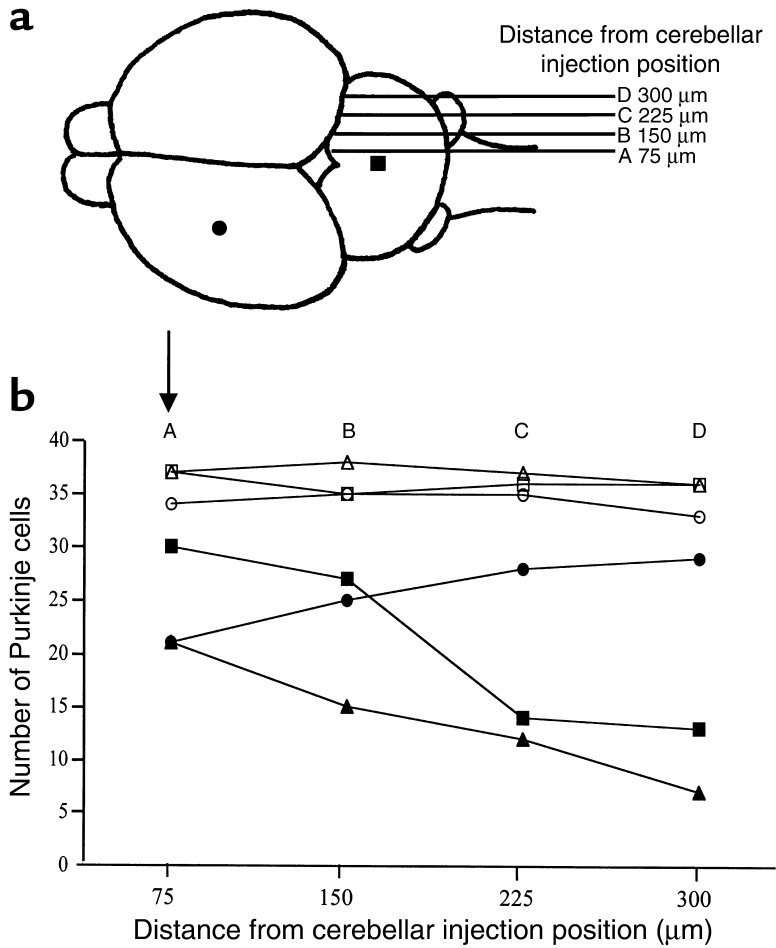

transplantation studies was due to the presence of donor-derived cells in the CNS that were releasing very low levels of ASM.

To further investigate this point, we used anti-human ASM antibodies to assess enzyme expression in brain sections. Notably, the surviving Purkinje cell neurons in treated animals had a strong positive signal for the human enzyme. There are two possible explanations for this result: (a) the retrovirally expressed human enzyme was secreted by donorderived cells present in the brain and taken up by the Purkinje cells, or (b) the donor cells themselves directly differentiated into Purkinje cells. Since the donor cells were derived from GFP transgenic mice, theoretically this question could be answered using a fluorescent detection system to colocalize the human ASM and GFP signals. However, when we used fluorescent

\section{Figure 7}

Purkinje cell survival in transplanted ASMKO mice is a function of distance from the cerebellar injection site. (a) Schematic depiction of a mouse brain showing the location of the hippocampal (filled circle) and cerebellar (filled square) injection sites. A-D indicate the location of sections used for the quantification shown in b (75-300 $\mu \mathrm{m}$ from the cerebellar injection site). The data shown in Figures 5 and 6 are from position A, indicated by the arrow in $\mathbf{b}$. Quantification was performed as described in Methods. Open symbols, normal animals; filled symbols, treated ASMKO animals; circles, 4 weeks after transplant; squares, 12 weeks after transplant; triangles, 24 weeks after transplant.

detection systems to label the anti-human ASM antibodies, the background signals were consistently too high to provide conclusive results.

We next sought to correlate the presence of human ASM in these cerebellar sections with a reduction in sphingomyelin content. Although our earlier histological analysis had shown markedly reduced vacuolization in the brains of treated mice, these studies did not reveal whether the specific lipid degraded by ASM (i.e., sphingomyelin) was reduced. We therefore used the sphingomyelin-specific binding protein, lysenin, to identify this lipid in brain sections, and found a very marked reduction in the treated mouse when compared with untreated ASMKO controls. This reduction was not limited to Purkinje cells, indicating that the expressed enzyme was probably secreted from donor cells and taken up by multiple neural cell types. Together these results indicate that functional ASM was clearly present in the brains of the treated animals and was likely responsible for the positive clinical and pathological effects we observed.

Similar to our previous bone marrow transplant and gene therapy experiments, the most notable histological effect in the CNS of the treated animals was on Purkinje cell survival, and this was directly related to improved cerebellar function. Although the explanation for this observation is not completely understood, recent studies have suggested that ceramide is a trophic factor required for Purkinje cell survival (27). Therefore, one could hypothesize that in NPD animals, the absence of

\section{Figure 8}

Expression of human ASM and depletion of sphingomyelin in the brains of transplanted ASMKO mice. The sections shown were taken from 4-month-old animals, $75 \mu \mathrm{m}$ from the cerebellar injection site. (a) Anti-human ASM antibodies were used to detect the retrovirally expressed enzyme in cerebellar sections of normal, untreated ASMKO, and treated ASMKO animals. Note that a strong positive (brown) signal was seen in Purkinje cells from the treated animals. No signal was seen in Purkinje cells from normal animals, demonstrating the specificity of the reaction. (b) The sphingomyelin-specific binding protein, lysenin, was used to detect this lipid in cerebellar sections from normal, untreated ASMKO, and treated ASMKO animals. Note that the sections from untreated ASMKO animals had a very strong lysenin signal compared with normal or treated ASMKO animals.
Treated ASMKO

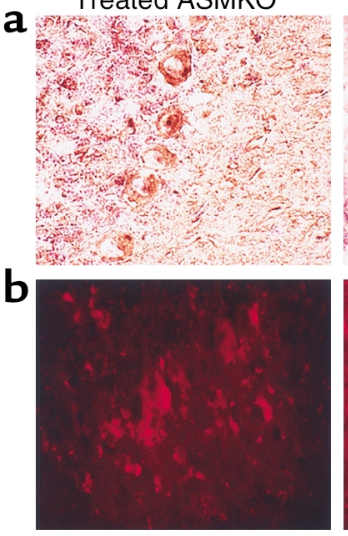

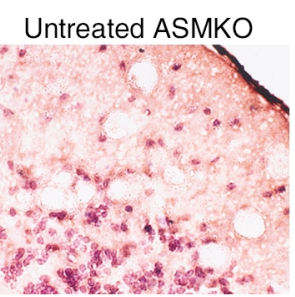

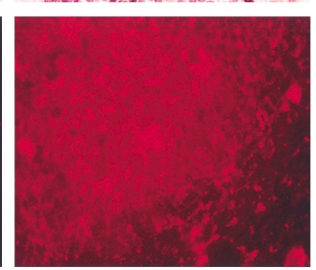

Normal
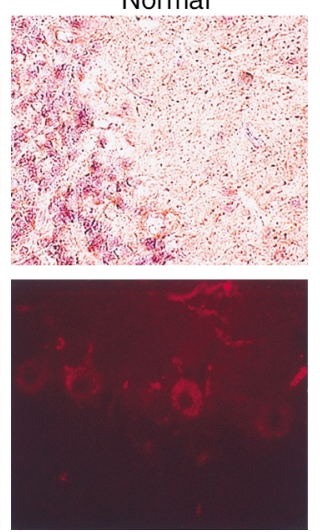
ASM activity in Purkinje cell neurons depletes pools of intracellular ceramide and negatively influences survival of this cell type. In the treated mice, ASM released from the transplanted MSCs may be taken up by Purkinje cells and replenish this ceramide pool. Cell culture studies are underway to evaluate ceramide production and ASM uptake by normal and NPD Purkinje cells. Other explanations that we cannot completely rule out are that the transplanted bone marrow cells might be providing some other trophic factor required for Purkinje cell survival, or that the transplanted cells are themselves differentiating into Purkinje cell neurons, as mentioned above. However, the results of a recent transgenic mouse experiment in which ASM was the only gene introduced onto the ASMKO background supports the notion that the clinical effect is likely related to the amount of functional ASM (or some other trophic factor) released by the transplanted cells, rather than their direct differentiation into Purkinje cells (28). In these animals, the Purkinje cell phenotype, cerebellar function, and life span were completely corrected when residual ASM activities of about $8 \%$ of normal were achieved in the CNS. Again, cell culture experiments are currently underway to directly address the issue of how much ASM activity is required for the survival of ASMKO Purkinje cells.

While the transplant results we present here are very encouraging, particularly given the very low levels of ASM activity in the CNS, they represented only partial correction of the neurological phenotype, and the animals still died prematurely. The most likely explanation for this is that we did not reconstitute enough ASM activity in the CNS for a more complete effect. This is probably due to the very small numbers of cells that were injected $(100,000)$ and the fact that these cells do not undergo much proliferation after transplantation. Indeed, we observed a general depletion of these cells over time in the transplanted ASMKO brains (data not shown). This was likely due to immunological rejection of the transplanted cells by the host animals, since (a) the donor cells were derived from GFP-expressing animals, (b) the genetic backgrounds and sex of the donor and host animals were different, and (c) the donor cells were overexpressing and releasing human ASM. The fact that we observed significant outcome differences between sex-matched and sex-mismatched transplants supports this notion.

To improve on the results reported here and obtain more ASM-expressing MSCs in the brain following transplantation, several strategies can be considered. First, and most straightforward, efforts can be made to reduce immunological rejection of the transplanted cells by using syngeneic, sex-matched donors. In the current study we wanted to use the GFP marker system to identify and map the MSCs after transplantation. However, these cells express foreign antigens that can elicit immune responses in the host animals. In addition, the donor cells were transduced with a human ASM retroviral vector. This could be modified in the future to use a vector that expresses murine ASM. It is important to recognize that in a human transplant setting, many of these immunological concerns should not be relevant since we would be using autologous cells from the affected patient for the transplant procedure. However, for these "proof of principle" experiments in the murine system they were clearly problematic.

In addition to minimizing immunological rejection of the donor cells, we could also use enriched populations of MSCs that might have greater proliferative capacity in the CNS, and/or adopt cell culture conditions that enhance proliferation. This may be necessary in the mouse model, since the number of cells we can transplant is very small. However, proliferating cells could cause tumors after transplantation, and this would need to be carefully evaluated. Finally, we could combine this approach with other approaches that can deliver ASM into the CNS. The most obvious strategy is to combine the intracerebral transplants with bone marrow transplantation, since the same source of cells (i.e., bone marrow) could be used for both procedures. In addition, we have already found that bone marrow transplantation effectively prevents the occurrence of visceral disease in these animals and also has partial effects on the CNS (4). Thus, the two procedures may be synergistic.

In conclusion, we have shown that the transplantation of very small numbers of ASM-expressing MSCs directly into the brains of NPD mice can elicit significant effects on the progression of their neurological disease and life span. This is the first report of this approach for any neurological lysosomal disease, and we believe it is an approach with direct clinical application. While similar intracerebral transplant studies have been carried out in other lysosomal disease models using cultured fibroblasts or neural stem cells $(29,30)$, the fact that autologous, bone marrow-derived MSCs can be easily obtained from patients and have excellent migratory capacity in the CNS makes them prime candidates for future therapeutic evaluation. However, the long-term survival of these cells in the CNS under conditions in which immunological rejection of the transplanted cells would be minimized remains to be determined. Future efforts will be directed toward improving on the results reported here in order to achieve a more complete CNS effect.

\section{Acknowledgments}

This research was supported by NIH grant HD-28607 and a generous donation from the Shulsky Foundation. Janet Carter is a Medical Research Council (United Kingdom) Clinician Scientist Fellow.

\footnotetext{
1. Schuchman, E.H., and Desnick, R.J. 2001. Niemann-Pick disease type A and B: acid-sphingomyelinase deficiencies. In The metabolic and molecular bases of inherited disease. C.R. Scriver, A.L. Beaudet, W.S. Sly, and D. Valle, editors. McGraw-Hill. New York, New York, USA. 3589-3610.

2. Horinouchi, K., et al. 1995. Acid sphingomyelinase deficient mice: a model of types A and B Niemann-Pick disease. Nat. Genet. 10:288-293.

3. Otterbach, B., and Stoffel, W. 1995. Acid sphingomyelinase-deficient mice mimic the neurovisceral form of human lysosomal storage disease (Niemann-Pick disease). Cell. 81:1053-1061.

4. Miranda, S.P.R., et al. 1998. Biochemical, pathologic and clinical
} 
response to transplantation of normal bone marrow cells into acidsphingomyelinase-deficient mice. Transplantation. 65:884-892.

5. Miranda, S.P.R., et al. 2000. Hematopoietic stem cell gene therapy leads to marked visceral organ improvements and a delayed onset of neurological abnormalities in the acid sphingomyelinase deficient mouse model of Niemann-Pick disease. Gene Ther. 7:1768-1776.

6. Prockop, D. 1997. Marrow stromal cells as stem cells for nonhematopoietic tissues. Science. 276:71-74.

7. Pereira, R.F.P., et al. 1998. Marrow stromal cells as a source of progenitor cells for nonhematopoietic tissues in transgenic mice with a phenotype of osteogenesis imperfecta. Proc. Natl. Acad. Sci. USA. 95:1142-1147.

8. Priller, J., et al. 2001. Neogenesis of cerebellar Purkinje neurons from gene-marked bone marrow cells in vivo. J. Cell Biol. 26:733-738.

9. Azizi, S.A., Stokes, D., Augelli, B.J., Digirolamo, C., and Prockop, D.J. 1998. Engraftment and migration of human bone marrow stromal cells implanted in the brains of albino rats-similarities to astrocyte grafts. Proc. Natl. Acad. Sci. USA. 95:3908-3913.

10. Kopen, G.C., Prockop, D.J., and Phinney, D.G. 1999. Marrow stromal cells migrate throughout the forebrain and cerebellum and they differentiate into astrocytes after injection into neonatal mouse brains. Proc. Natl. Acad. Sci. USA. 96:10711-10716.

11. Schwarz, E.J., Alexander, G.M., Prockop, D.J., and Azizi, S.A. 1999. Multipotential marrow stromal cells transduced to produce L-DOPA: engraftment in a rat model of Parkinson's disease. Hum. Gene Ther. 10:2539-2549.

12. Friedenstein, A.J., Derisglasova, U.F., Kulagina, N.N., Panasuk, A.F., and Keiliss-Borok, I.V. 1974. Precursors for fibroblasts in different populations of hematopoietic cells as detected by the in vitro colony assay method. Exp. Hematol. 2:83-92.

13. Yeyati, P.L., et al. 1995. Fluorescence-based selection of retrovirally transduced cells in the absence of a marker gene: direct selection of transduced type B Niemann-Pick disease cells and evidence for bystander correction. Hum. Gene Ther. 6:975-983.

14. He, X., et al. 1999. Characterization of human acid sphingomyelinase purified from the media of overexpressing Chinese hamster ovary cells. Biochim. Biophys. Acta. 1432:251-264.

15. Yamaji, A., et al. 1998. Lysenin, a novel sphingomyelin-specific binding protein. J. Biol. Chem. 273:5300-5306.

16. Dunham, N.W., and Miya, T.S. 1957. A note on a simple apparatus for detecting neurological deficit in rats and mice. J. Am. Pharm. Assoc. 46:XLVI.

17. Jones, B.J., and Roberts, D.J. 1968. The quantitative measurement of motor incoordination in naive mice using an accelerating rotarod.
J. Pharm. Pharmacol. 20:302-304.

18. Wells, J., Vietje, B.P., Wells, D.G., and Dunn, G.E. 1988. Cell-sized microspheres in the hippocampus show cleavage planes and passive displacement. Brain Res. Bull. 21:601-605.

19. Fricker, R.A., et al. 1999. Site-specific migration and neuronal differentiation of human neural progenitor cells after transplantation in the adult rat brain. J. Neurosci. 19:5990-6005.

20. Woodbury, D., Schwarz, E.J., Prockop, D.J., and Black, I.B. 2000. Adult rat and human bone marrow stromal cells differentiate into neurons. J. Neurosci. Res. 61:364-370.

21. Fukunaga, A., Uchida, K., Hara, K., Kuroshima, Y., and Kawase, T. 1999. Differentiation and angiogenesis of central nervous system stem cells implanted with mesenchyme into ischemic rat brain. Cell Transplant. 8:435-441.

22. Leboy, P.S., Beresford, J.N., Devlin, C., and Owen, M.E. 1990. Dexamethasone induction of osteoblast mRNAs in rat marrow stromal cell cultures. J. Cell. Physiol. 146:370-378.

23. Johnstone, B., Hering, T.M., Caplan, A.I., Goldberg, V.I., and Jung, Y.U. 1998. In vitro chondrogenesis of bone marrow-derived mesenchymal stem cells. Exp. Cell Res. 238:265-272.

24. Bennett, J.H., Joyner, C.J., Triffitt, J.T., and Owen, M.E. 1991. Adipocytic cells cultured from marrow have osteogenic potential. J. Cell Sci. 99:131-139.

25. Wakitani, S., Saito, T., and Caplan, A.I. 1995. Myogenic cells derived from rat bone marrow mesenchymal cells exposed to 5-azacytidine. Muscle Nerve. 18:1417-1426.

26. Miranda, S.R.P., et al. 2000. Infusion of recombinant human acid sphingomyelinase into Niemann-Pick disease mice leads to visceral, but not neurological, correction of the pathophysiology. FASEB J. 14:1988-1995.

27. Furuya, S., et al. 2000. L-serine and glycine serve as major astrogliaderived trophic factors for cerebellar Purkinje neurons. Proc. Natl. Acad. Sci. USA. 97:11528-11533.

28. Marathe, S., et al. 2000. Creation of a mouse model for non-neurological (type B) Niemann-Pick disease by stable, low level expression of lysosomal sphingomyelinase in the absence of secretory sphingomyelinase: relationship between brain intra-lysosomal enzyme activity and central nervous system function. Hum. Mol. Genet. 9:1967-1976.

29. Taylor, R.M., and Wolfe, J.H. 1997. Decreased lysosomal storage in the adult MPS VII mouse brain in the vicinity of grafts of retroviral vectorcorrected fibroblasts secreting high levels of beta-glucuronidase. Nat. Med. 3:771-774.

30. Torchiana, E., et al. 1998. Retroviral-mediated transfer of the galactocerebrosidase gene in neural progenitor cells. Neuroreport. 9:3823-3827. 